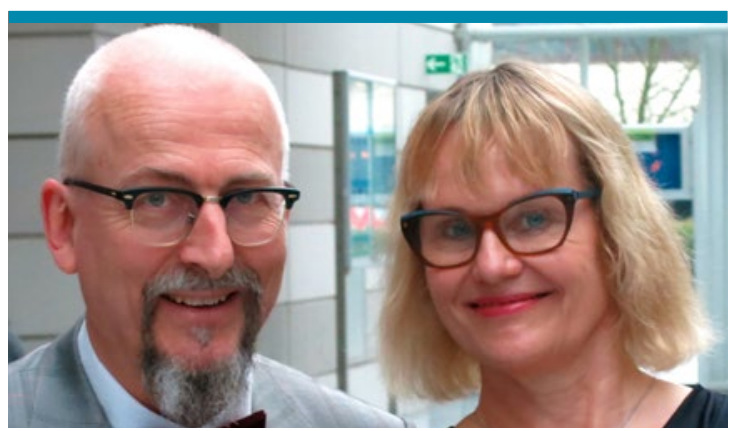

"Berlin gilt als rau, aber herzlich - der 11. Deutsche Allergiekongress wird so ähnlich: aktuelle Allergiefortbildung für Klinik und Praxis plus lebendige Diskussion. Gönnen Sie sich den Hauptstadtbesuch kurz vor dem Tag der Deutschen Einheit."

Präsidiales Duo für Berlin: Prof. Dr. Susanne Lau, Charité - Universitätsmedizin Berlin und PD Dr. Jörg Kleine-Tebbe, Allergie- und Asthma-Zentrum Westend, Berlin

\title{
Moderne Allergologie - die Zukunft ist schon da!
}

W enige Wochen vor dem 11. Deutschen Allergiekongress in Berlin sind die Weichen gestellt. Verpassen Sie den fahrenden Zug nicht: Moderne Allergologie - Konzepte für die Zukunft. Dabei geht es weniger um Pläne fürs nächste Jahrzehnt, sondern um echte Fortschritte, die sich schon jetzt in Klinik und Praxis umsetzen lassen.

Die Allergologie befindet sich im Umbruch. Wissenschaftliche Neuigkeiten beleben das Querschnittsfach an allen Enden. Dies betrifft auch die klinische Allergologie mit aktuellen Ansätzen zu Prävention, Diagnostik und Therapie. Molekulare Allergologie, Toleranzvehikel und Biologicals klopfen lautstark an die Tür. Manche Routine in Klinik und Praxis hat schlicht ausgedient. Die diagnostische Pyramide wird derzeit kräftig durchgeschüttelt und die Immuntherapie auf den Prüfstand gestellt.

Andererseits zeigt die praktische Allergologie echte Mangelerscheinungen: Weniger Diagnostika für die Haut bei Sofort- und Spättypallergie, Monopoly bei den Epikutantest-Reagenzien, Gängelei durch EBMUnfug, weniger Individualrezepturen und vor allen

\section{Deutscher Allergiekongress auf einen Blick}

\section{Veranstalter}

- Deutsche Gesellschaft für Allergologie und klinische Immunologie (DGAKI)

- Ärzteverband Deutscher Allergologen (AeDA)

_ Gesellschaft für Pädiatrische Allergologie und Umweltmedizin (GPA)

\section{Kongresstermin und-Ort}

29. September bis 1. Oktober 2016 im Berlin Congress Center

\section{Anmeldung und Information}

Weitere Infos gibt es unter www.allergiekongress.de. Die Anmeldung ist online möglich oder mit nebenstehendem Anmeldeformular.
Dingen unzureichende Vergütung für eine „sprechende Medizin“. Viele Kollegen haben - wie die Briten mit ihrem Brexit - die Abkehr vom Fach Allergologie, den AllergoExit, längst vollzogen. Die Politik hat das erkannt. Initiativen zur gesicherten Information betroffener Allergiker sollen retten, was an ärztlicher Kompetenz und Beratung zu fehlen beginnt.

Hier ist Fortbildung gefragt: Allergieanamnesen in 5 Min., Anaphylaxie-Aufklärung in $2 \mathrm{Min}$., Nahrungsmittelallergieberatung durch rhythmisches Schulterheben - Schluss mit lustig, denn es gibt sie, die zielführenden Fragen, die rationale Diagnostik und die individuellen Therapieansätze für eine pragmatische lösungsorientierte Allergologie. Diskutieren Sie mit Profis neue Möglichkeiten des Allergiemanagements. Erweitern Sie Ihr Wissen und lernen Sie das Handwerkszeug einer modernen Allergologie kennen. Die Formate haben sich bewährt:

-AllergoCompact: Die wichtigsten Themen der Allergologie für Sie extrahiert

_AllergoActive: Interaktive Seminare, praktische Übungen, konkrete Anleitungen

-AllergoArena: Pro-und-Con-Debatten mit TEDAbstimmungen: Sie sind gefragt!

-AllergoConnect: Geschwistersymposien mit anderen Gesellschaften

_AllergoPlenum: Übersichtsvorträge vom Feinsten zu aktuellen Themen

_Curriculum der Allergologie: Praktische Diagnostik und Therapie

Nutzen Sie die wichtigste Allergiefortbildung im Lande, den 11. Deutschen Allergiekongress 2016. Wir freuen uns, Sie in Berlin zu begrüßen!

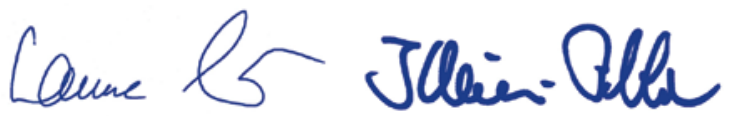

